\title{
Pre-clerkship teaching: Are we missing an opportunity?
}

\author{
Viren N. Naik, MD, MEd, MBA, FRCPC
}

Received: 30 September 2016/Revised: 5 October 2016/ Accepted: 14 October 2016/Published online: 24 October 2016

(C) Canadian Anesthesiologists' Society 2016

Before you read this article, close your eyes and reflect on medical school and that sentinel moment when you decided to pursue a career in anesthesiology. It is certainly easy to remember the influential mentors and coaches from residency, but after years in practice, try to recall that moment in undergraduate medicine when your career thoughts turned to this specialty and changed your life forever.

In this month's issue of the Journal, Hamlin et al. report on their survey regarding the participation of Canadian departments of anesthesiology in undergraduate education. The authors focus their report on the pre-clerkship curriculum $^{1}$ and express their concern that the absolute volume of teaching to our year 1 and 2 medical students falls far below the ideal. The authors contend that we are jeopardizing the future of the specialty by failing to capture the early interest and imagination of the best and brightest students and, in so doing, that we are hindering the development of future educators, researchers, and leaders in our specialty.

Critics will question the validity of the survey data by highlighting an incomplete return from every stakeholder. They may wonder whether the data truly accounted for all the interactions, or they may ask why our traditional role in one-to-one teaching during clerkship should be discounted. Indeed, many readers may experience the Kübler-Ross stages of grief when reviewing this manuscript, ${ }^{2}$ namely, denial (there is no problem), anger (the data are wrong), bargaining (different metrics are needed), depression (it's

\section{N. Naik, MD, MEd, MBA, FRCPC (ه)}

Department of Anesthesiology and Pain Medicine, University of Ottawa, 1053 Carling Ave, Room AM 48, Ottawa, ON K1Y 4E9, Canada

e-mail: vnaik@toh.ca harder for anesthesiology), and finally acceptance (a change needs to occur). In acceptance, our specialty will acknowledge that, while measuring and interpreting these data may be imperfect, they clearly put forward a signal for discussion.

It is noteworthy that the present work is a follow-up to the 2001 survey that first raised concern regarding our specialty's engagement in the undergraduate curriculum. ${ }^{3}$ Optimists will anchor themselves in the significant improvement in teaching hours per anesthesiologist compared with the previous survey. Pragmatists will argue that our specialty is unique in terms of service obligations, and therefore, the results are "not that bad".

So how important is a specialty's involvement in the undergraduate curriculum? What influence does exposure to a specialist have on the ultimate selection of a specialty career? Is "not bad" the same as "good enough", or should we be striving for "better" and "great"? Clearly, these questions are complex, and it would be inappropriate to suggest that exposure is the single most important variable that contributes to career selection amongst medical students. What we do know is that most medical students move toward their preferred specialty well before their clerkship rotations. ${ }^{4,5}$ This should come as little surprise to recent graduates, as competition for preferred residency positions has become more intense. To compound this challenge, the application process for clerkship electives (still the best opportunity for medical students to be noticed for selection to a program) has become more standardized and centralized in recent years in order to improve fairness. ${ }^{6}$ The unintended consequence of this process is that medical students need to be even more certain about their elective requests well in advance of their clerkship years. Medical students are aligning themselves towards a 
specialty earlier and earlier, and with respect to our specialty, they crave more information and interaction with anesthesiologists. ${ }^{7}$ In the absence of adequate exposure to anesthesiologists in these formative pre-clerkship years, their impressions will be influenced by incomplete or inaccurate information from our colleagues in other specialties, or worse, the media and popular culture. ${ }^{7}$

As mentioned by Hamlin et al., anesthesiologists are well positioned to instruct at the undergraduate level. ${ }^{1}$ Our broad education crosses many diseases, illnesses, and treatments. Much of our knowledge and many of our skills cross different specialties and blend many medical and surgical competencies. Our comprehensive understanding of pharmacology and physiology is a definite asset to the novice learner in medicine, and our involvement would allow students to link theoretical content to clinical relevance. Also important, our non-medical expert competencies that facilitate management and teamwork in the operating theatre and perioperative clinics can be leveraged to integrate CanMEDS in the undergraduate curriculum where appropriate. ${ }^{8}$ Finally, simulation-based education was born out of our specialty. ${ }^{9}$ The $21^{\text {st }}$ century has seen and will see a greater reliance on simulation to deliver "hands on" active learning of both medical expert and intrinsic CanMEDS roles in a patient-safe environment. ${ }^{10}$ With our pedigree in theatre-based simulation, who better than anesthesiologists to offer sound leadership and frontline teaching on how to integrate this resource-intensive tool into the undergraduate curriculum. It is somewhat paradoxical that our commitment and opportunity to teach in the undergraduate programs are questioned when many of our colleagues are involved in the highest levels of leadership in faculties of medicine across this country, including the undergraduate dean position at several institutions. Opportunities for anesthesiologists exist, but after historically limited engagement, it is little wonder why recruiters for undergraduate teaching may seek alternative subject matter experts. Opportunities do exist, but now we may need to ask.

The opportunity cost of participating in any formal teaching is a barrier for any specialty. It is often easier for departments to justify investment in postgraduate teaching, as departments are more directly accountable to deliver these requirements for accreditation. With respect to undergraduate education, particularly during preclerkship, accountability for the delivery of the curriculum is rarely isolated to a single department, and any return on investment is much less tangible. Even when an academic institution offers some remuneration, the income fails to match that of clinical earnings. It may be a cliché, but it is still worth mentioning that the rewards of teaching go beyond remuneration. Indeed, the literature supports that the intrinsic rewards of teaching, including gratification from watching well-educated and motivated medical students master knowledge or a skill, are important contributors to career satisfaction. Another benefit for many clinicians is a renewed interest in medicine after years in practice by providing a powerful motivator for lifelong learning in the delivery of an evidence-based curriculum. ${ }^{10}$ Finally, engaging in teaching creates opportunities for academic advancement and peer recognition for education excellence. This benefits not only the individual but also the entire department for their demonstrable support.

No one is denying that the logistics of participating in pre-clerkship teaching sessions are different for anesthesiologists than for physicians in other specialties. Our services are scheduled more rigidly and require a higher degree of direct staff physician involvement in the delivery of patient care. Before debating "how" we manage conflicting priorities, we need to focus on "why" we need to value undergraduate education. Once we can agree on the "why", the "how" will develop through practice plans, anesthesia care teams, graded resident supervision, or other innovative academic models. I encourage you to use this issue of the Journal as an excellent starting point for these important discussions. While in those discussions, consider how different your life would be had there been no anesthesiologists to meet in medical school, igniting your passion for the specialty.

\section{L'enseignement pré-stage clinique: Passons-nous à côté d'une occasion?}

Avant de lire cet article, fermez vos yeux un instant; souvenez-vous de votre passage à l'école de médecine : rappelez-vous de ce moment marquant où vous avez décidé de poursuivre une carrière en anesthésiologie. Il vous est probablement facile de vous rappeler les mentors et les personnes qui vous ont influencé pendant votre résidence; toutefois, après plusieurs années de pratique, essayez de vous remémorer l'instant précis, au cours de vos études de médecine de premier cycle, où vous avez considéré cette spécialité comme choix de carrière et que votre vie a changé pour toujours.

Dans ce numéro du Journal, Hamlin et coll. rendent compte des résultats de leur questionnaire portant sur la participation des départements canadiens d'anesthésiologie à la formation de premier cycle. Les auteurs se sont concentrés sur le cursus précédant le stage clinique ${ }^{1}$ de l'externat et ils nous font part de leurs inquiétudes : en 
effet, le volume absolu d'enseignement à nos étudiants de première et deuxième années de médecine est bien en-deçà du volume idéal. Selon les auteurs, nous sommes en train de mettre en péril l'avenir-même de notre spécialité en n'attisant pas assez tôt l'intérêt et l'imagination des meilleurs étudiants et, par conséquent, nous nuisons à l'essor de futurs enseignants, chercheurs et chefs de file dans notre spécialité.

Les esprits critiques ne manqueront pas de remettre en question la validité des données du questionnaire en montrant du doigt les rétroactions incomplètes de toutes les parties concernées. Ces critiques peuvent se demander si les données tirées du questionnaire tiennent véritablement compte de toutes les interactions, ou pourquoi notre rôle traditionnel d'enseignement individuel pendant le stage clinique de l'externat devrait être négligé. En fait, plusieurs lecteurs pourraient, en lisant ce manuscrit, traverser les diverses phases du deuil selon Kübler-Ross ${ }^{2}$ : d'abord le déni (il n'y a pas de problème), puis la colère (les données sont erronées), suivie par la négociation (il faut utiliser d'autres critères), puis la dépression (c'est plus difficile en anesthésiologie), et finalement l'acceptation (il faut que ça change). Au cours de la phase d'acceptation, notre spécialité reconnaîtra que, bien que la mesure et l'interprétation de ces données puissent être imparfaites, elles ouvrent clairement la porte à une discussion plus poussée.

Il faut souligner que les travaux présentés ici sont le suivi d'un questionnaire réalisé en 2001 qui avait déjà tiré la sonnette d'alarme quant à l'implication de notre spécialité dans le programme de formation de premier cycle. ${ }^{3}$ Les optimistes se féliciteront des progrès importants en ce qui touche au nombre d'heures d'enseignement par anesthésiologiste par rapport au questionnaire de 2001. Les pragmatistes, quant à eux, soutiendront que notre spécialité est unique en termes d'obligations de service et que, tout compte fait, ces résultats ne sont «pas si mauvais ».

Dès lors, quelle est l'importance de l'implication d'une spécialité dans le programme de formation de premier cycle? Quelle influence peut avoir le contact avec un spécialiste sur la sélection finale d'un choix de carrière dans une spécialité donnée? Est-ce que "pas mauvais» signifie «suffisamment bon», ou devrions-nous viser quelque chose de «mieux », voire «l'excellence »? Ces questions n'ont certes aucune réponse facile, et loin de nous l'idée de suggérer que l'exposition à la spécialité est la variable la plus importante qui influence le choix de carrière des étudiants en médecine. Ce que nous savons, en revanche, c'est que la plupart de ces étudiants s'orientent vers leur spécialité de prédilection bien avant de commencer leurs stages cliniques d'externat. ${ }^{4,5}$ Ceci est probablement très peu surprenant pour les jeunes diplômés, au vu de l'intensification de la compétition pour les postes de résidence prisés. Pour compliquer les choses, depuis quelques années, le processus de postulation pour les stages à option de l'externat clinique - qui demeurent la meilleure façon, pour les étudiants en médecine, de se démarquer pour être ensuite retenus pour un programme de résidence - a été normalisé et centralisé afin d'en améliorer l'impartialité. ${ }^{6}$ La conséquence involontaire de ce processus est que les étudiants en médecine doivent être encore plus certains quant à leurs demandes de stages à option bien avant que leurs stages cliniques ne commencent. Les étudiants en médecine s'orientent de plus en plus tôt vers une spécialité et, en ce qui touche à la nôtre, ils aimeraient obtenir davantage d'informations et d'interactions avec des anesthésiologistes. ${ }^{7}$ En l'absence d'une exposition adéquate aux anesthésiologistes au cours des années d'apprentissage précédant l'externat clinique, leurs impressions seront influencées par des informations incomplètes ou inexactes glanées auprès de nos collègues d'autres spécialités, ou pire, des médias et de la culture populaire. $^{7}$

Comme le mentionnent Hamlin et coll., les anesthésiologistes occupent une position privilégiée pour enseigner au niveau du premier cycle. ${ }^{1}$ Notre formation diversifiée recoupe de multiples maladies, conditions et traitements, et bon nombre de nos connaissances et de nos compétences recoupent plusieurs spécialités et marient des compétences médicales et chirurgicales. Notre compréhension approfondie de la pharmacologie et de la physiologie constitue indubitablement un atout pour tout étudiant en médecine novice, et notre implication permettrait aux étudiants de faire le lien entre un contenu théorique et sa pertinence clinique. Qui plus est, nos compétences et de notre expertise non médicales pourraient également être mises à profit, car elles facilitent la prise en charge et le travail d'équipe en salle d'opération et dans les cliniques périopératoires; on pourrait les utiliser pour incorporer les compétences CanMEDS pertinentes dans le programme de cours du premier cycle. ${ }^{8}$ Enfin, la formation basée sur la simulation est littéralement née de notre spécialité. ${ }^{9}$ Le $21^{\mathrm{e}}$ siècle a vu et verra une dépendance croissante envers la simulation, car cet outil permet d'offrir une forme d'apprentissage actif et concret des rôles d'expert médical tout autant que des rôles intrinsèques CanMEDS, le tout dans un environnement sécuritaire pour le patient. ${ }^{10}$ Étant donné notre expérience en matière de simulation en salle d'opération, qui est mieux placé que nous, les anesthésiologistes, pour offrir un leadership éclairé et un enseignement de pointe quant à la façon d'incorporer cet outil avide de ressources dans le cursus de premier cycle? Il est quelque peu paradoxal que notre implication et notre occasion d'enseigner au niveau du premier cycle soient remis en question : en effet, bon nombre de nos collègues s'impliquent aux plus hauts 
niveaux de direction et de gestion dans les facultés de médecine partout au pays, notamment en tenant le poste de doyen aux études de premier cycle dans plusieurs institutions. Les occasions sont donc bien réelles pour les anesthésiologistes - mais, après notre implication historiquement limitée, il n'est pas surprenant que les recruteurs pour l'enseignement de premier cycle cherchent d'autres experts. Les occasions existent, certes, mais c'est désormais à notre tour d'être proactif et de poser la question.

Peu importe la spécialité, le manque à gagner subi lorsqu'on participe à toute forme d'enseignement formel constitue un obstacle. Il est souvent plus facile pour un département de justifier un investissement dans l'enseignement supérieur, étant donné que l'imputabilité des départements est plus directe quant il s'agit de remplir ces exigences pour obtenir leur certification. En revanche, quand il est question de la formation de premier cycle, particulièrement avant l'externat clinique, l'imputabilité pour la prestation des cours affecte rarement un seul département, et tout retour sur investissement est bien moins tangible. Même lorsqu'une institution universitaire offre une forme de rémunération, le salaire est bien loin du salaire clinique. C'est peut-être un cliché, mais il convient tout de même de rappeler que les retours de l'enseignement vont bien au-delà d'une simple rémunération. En effet, selon la littérature, les récompenses intrinsèques de l'enseignement, notamment la gratification de voir des étudiants de médecine bien éduqués et motivés maîtriser des connaissances ou des compétences, constituent d'importants facteurs qui contribuent à la satisfaction professionnelle. Un autre avantage, pour plusieurs cliniciens, réside dans l'intérêt nouvellement attisé pour la médecine après des années de pratique, en proposant un élément de motivation puissant pour une éducation permanente dans la transmission d'un cursus fondé sur des données probantes. ${ }^{10}$ Enfin, l'implication dans l'enseignement crée des occasions de réussite universitaire et de reconnaissance des pairs pour l'excellence de la formation. Ceci bénéficie non seulement l'individu, mais le département dans son intégrité grâce à son soutien apparent.

Nous sommes tous d'accord que la logistique nécessaire à la participation a des séances d'enseignement précédant le stage clinique est différente pour les anesthésiologistes que pour les médecins d'autres spécialités. Nos services sont programmés de façon plus rigoureuse et nécessitent un niveau plus important d'implication directe du médecin en charge pour la prestation des soins aux patients. Avant de discuter de « comment » gérer ces priorités contradictoires, nous devons nous concentrer sur «pourquoi » il est crucial que nous accordions de l'importance à la formation de premier cycle. Une fois que nous aurons convenu de cette raison, nous trouverons des façons de l'intégrer à notre pratique, que ce soit via des programmes de pratique, des équipes de soins anesthésiques, de la supervision de résidents avec évaluation, ou d'autres modèles universitaires innovants. Je vous invite à utiliser ce numéro du Journal comme point de départ pour initier ces discussions essentielles au sein de votre pratique. Au cours de vos discussions, rappelez-vous de l'anesthésiologiste ou des anesthésiologistes que vous avez rencontrés à l'école de médecine, de ceux et celles qui ont éveillé votre passion pour cette spécialité, et pensez à quel point votre vie aurait été différente s'ils n'avaient pas été là et que vous n'aviez pas eu l'occasion de les rencontrer.

Conflicts of interest None declared.

Editorial responsibility This submission was handled by Dr. Philip M. Jones, Associate Editor, Canadian Journal of Anesthesia.

\section{Conflit d'intérêt Aucun.}

Responsabilité éditoriale Cet article a été traité par Dr Philip M. Jones, rédacteur adjoint, Journal canadien d'anesthésie.

\section{References}

1. Hamlin $C$, Bhangu $K$, Villafranca A, et al. Participation of Canadian anesthesiology departments in undergraduate medical education. Can J Anesth 2017; 64. DOI: 10.1007/s12630-0160761-4.

2. Kübler-Ross E. On Death and Dying. NY: Macmillan Company; 1969 .

3. Brull R, Bradley JW. The role of anesthesiologists in Canadian undergraduate medical education. Can J Anesth 2001; 48: 14752.

4. Basco WT Jr, Reigart JR. When do medical students identify career-influencing physican role models? Acad Med 2001; 76: 380-2.

5. Hochberg MS, Billig J, Berman RS, et al. When surgeons decide to become surgeons: new opportunities for surgical education. Am J Surg 2014; 207: 194-200.

6. Association of Faculties of Medicine of Canada (AFMC). AFMC Student Portal, 2016. Available from URL: https://www. afmcstudentportal.ca/About (accessed October 2016).

7. Robertson EM, Henschke SJ, Friesen J, Tsui BC. Survey of preclerkship medical students' knowledge and perceptions of anesthesiology at one Canadian university. Can J Anesth 2015; 62: $550-2$.

8. Hodges BD, Albert M, Arweiler D, et al. The future of medical education: a Canadian environmental scan. Med Educ 2011; 45: 95-106.

9. Bradley $P$. The history of simulation in medical education and possible future directions. Med Educ 2006; 40: 254-62.

10. Gerrity MS, Pathman DE, Linzer M, et al. Career satisfaction and clinician-educators. The rewards and challenges of teaching. The Society of General Internal Medicine Career Satisfaction Study Group. J Gen Int Med 1997; 12(Suppl 2): S90-7. 